\title{
Terahertz Time-Domain Spectroscopy to Identify and Evaluate Anomer in Lactose
}

\author{
Satoshi Yamauchi ${ }^{1 *}$, Sakura Hatakeyam ${ }^{1}$, Yoh Imai $^{2}$, Masayoshi Tonouchi ${ }^{3}$ \\ ${ }^{1}$ Department of Biomolecular Functional Engineering, Ibaraki University, Hitachi, Japan \\ ${ }^{2}$ Department of Electric and Electronic Engineering, Ibaraki University, Hitachi, Japan \\ ${ }^{3}$ Institute of Laser Engineering, Osaka University, Osaka, Japan \\ Email: *ysatoshi@mx.ibaraki.ac.jp
}

Received November 9, 2013; revised December 2, 2013; accepted December 12, 2013

Copyright (C) 2013 Satoshi Yamauchi et al. This is an open access article distributed under the Creative Commons Attribution License, which permits unrestricted use, distribution, and reproduction in any medium, provided the original work is properly cited.

\begin{abstract}
Lactose powder consisting of $\alpha$-D-lactose monohydrate and anhydrous $\beta$-D-lactose was nondestructively and quantitatively evaluated by transmission-type Terahertz time-domain spectroscopy (THz-TDS). An absorption with peak at 39.7 $\mathrm{cm}^{-1}(1.19 \mathrm{THz})$ was assigned to be derived from anhydrous $\beta$-D-lactose, in addition to the absorptions due to $\alpha$-Dlactose monohydrate with peak at $17.1 \mathrm{~cm}^{-1}(0.53 \mathrm{THz})$ and $45.6 \mathrm{~cm}^{-1}(1.37 \mathrm{THz})$. After deconvolution of the spectra using Lorentzian, integrated intensities of the absorptions with peak at $39.7 \mathrm{~cm}^{-1}$ and $45.6 \mathrm{~cm}^{-1}$ were uniquely dependent on the weight composition ratio of the $\alpha$ - and $\beta$-lactose powder. As a result, the net molar-ratio of the $\alpha$ - and $\beta$-lactose in lactose powder could be precisely evaluated by the integrated intensity ratio. Further, anomer content in lactose powder extracted from lactose solution was evaluated and the refined and unrefined features were shown by the evaluation method.
\end{abstract}

Keywords: THz-TDS; Lactose-Powder; Anomer; $\alpha$-Lactose; $\beta$-Lactose

\section{Introduction}

Lactose comprising a glucose linked to a galactose abundantly present in milk of most mammals is an important disaccharide used in foods and pharmaceutical applications. Two anomers ( $\alpha$-lactose and $\beta$-lactose) commonly exist in the disaccharide powder. Evaluation and control of the ratio are important to use lactose in foods and drugs because the $\alpha$ - and $\beta$-form show significantly different physicochemical properties, i.e. solubility $(\beta$-form is more soluble than $\alpha$-form) and hardness ( $\alpha$-form is harder than $\beta$-form), and crystallized shape [1]. Commonly, lactose can be prepared as $\alpha$-lactose monohydrate and anhydrous $\beta$-lactose crystalline in addition to the amorphous phase of the $\alpha$ - and $\beta$-form mixture. The $\alpha$ and $\beta$-crystals are usually formed in supersaturated lactose solution, where $\alpha$-lactose can be crystallized with preventing the $\beta$-crystallization at room-temperature but the $\beta$-lactose is condensed above $93.5^{\circ} \mathrm{C}$ with extracting of the $\alpha$-crystal [2]. Amorphous lactose fabricated by freeze-drying or spray-drying is crystallized into several crystal forms such as $\alpha$-lactose monohydrate [3], anhy-

${ }^{*}$ Corresponding author. drous $\beta$-lactose [4] and anhydrous crystal with $\alpha$ - and $\beta$-lactose in molar ratios of 5:3 and 4:1 [5]. The crystallization which may enhance both physical and chemical deterioration [6] is dependent on the composition ratio, drying process, storage temperature, period and humidity [7], where the precise evaluation of crystallized $\alpha / \beta$-form ratio is so important to study the crystallization feature.

Commonly, the $\alpha$-lactose/ $\beta$-lactose ratio and the crystallization behavior are analyzed by thermogravimetric analysis (TGA) and differential scanning calorimetry (DSC) $[8,9]$, Raman spectroscopy [10], Fourier transform infrared spectroscopy (FTIR) [11], X-ray diffraction (XRD) $[7,12]$ etc. However, the TGA and DSC bring to destruction of sample, and the Raman spectroscopy, FTIR and XRD can evaluate in significantly thin region limited within dozens of micrometers. In contrast, THz-spectroscopy is expected to be useful for nondestructive characterization of materials with the thickness in millimeter-order because THz-electromagnetic wave in very-farinfrared region is notably absorbed in water but easily passed through most inorganic and organic materials comparing to UV-vis-infrared light. Further, it has been recognized that $\mathrm{THz}-\mathrm{absorptions}$ based on the intra-mo- 
lecular vibrations in organics and/or the inter-molecular dynamics incorporated hydrogen-bonding are significantly dependent on molecular- and crystal-structure as demonstrated for DL-alanine racemic compound comparing to D- and L-alanine [13], L-phenylalanine comparing to Ltyrosine [14], and three different retinal isomers with polyene chain [15]. Many monosaccharides and disaccharides are also interesting biomolecules for the THzspectroscopy since they show the spectral fingerprints with relatively narrow-bands in THz-region [16,17]. $\alpha$-Dlactose also shows the typical absorptions with narrowbands in THz-region below $3 \mathrm{THz}$ [18] as well as the other disaccharides. However, THz-spectroscopy has not been used to determine the anomer content because the absorption spectra derived from $\beta$-D-lactose has not been identified.

In this study, transmission-type $\mathrm{THz}$ time-domain spectroscopy (THz-TDS) is applied to characterize lactose. Firstly, we focus to identify THz-absorption due to $\beta$-Dlactose, then achieve quantitative evaluation of net molar ratio of $\alpha$ - and $\beta$-lactose in the mixtures using integrated intensity ratio of the absorptions due to $\alpha$-D-lactose and $\beta$-D-lactose.

\section{Experimental}

\subsection{THz-TDS System}

Transmission-type THz-TDS system as shown in Figure 1 was used to characterize lactose-powers. After a femto-second laser-light (peak wavelength at $782 \mathrm{~nm}$, half-width of $87 \mathrm{fs}$, repetition rate of $48 \mathrm{MHz}$ ) was split into a pump light and a probe light, the pump light chopped at $1 \mathrm{kHz}$ was focused and irradiated on a $\mathrm{THz}$ emitter consisting of a dipole antenna with $10 \mu \mathrm{m}$-gap space fabricated on LT-GaAs layer and attached on a hemispherical Si-lens, in which the antenna was biased at $10 \mathrm{~V}$ to generate transient current in pico-second order. The radiated THz-pulse through the Si-lens was focused

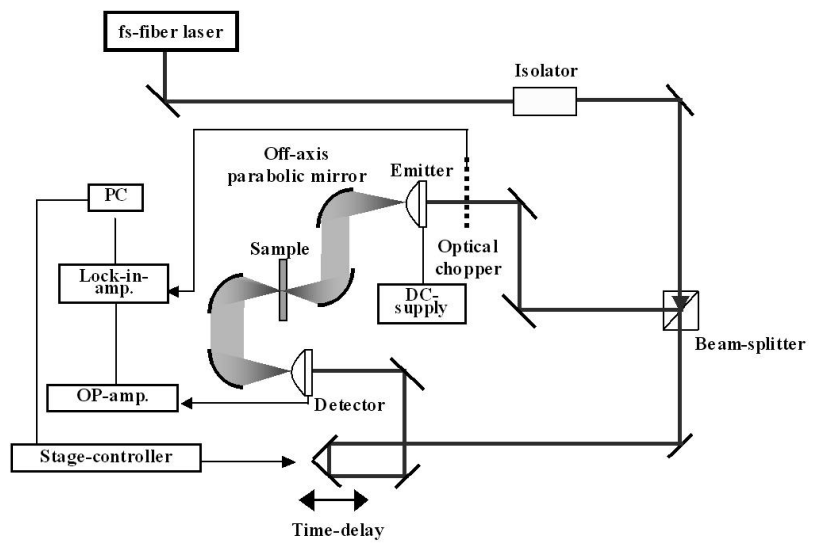

Figure 1. Schematic configuration of transmission-type $\mathbf{T H z}$ time-domain spectroscopy (THz-TDS) system. and normally incident to the sample using two off-axis parabolic metal mirrors. Then the THz-pulse passed through the sample was introduced to the detector with the same antenna configuration of the emitter by two off-axis parabolic mirrors and a hemispherical Si-lens. When the THz-pulse was introduced to the antenna on the detector, the probe laser light was simultaneously irradiated to detect the pulse by sampling technique, where time-delay of the probe light was controlled by a retroreflector and a micro-step stage controller with the step length of $1 \mu \mathrm{m}$. The sampling data were recorded in a PC after signal-amplification, lock-in noise reduction and $\mathrm{A} / \mathrm{D}$ conversion. The recorded pulse data was processed by a DFT after a Gaussian-window was superposed on the pulse data to remove aliasing. Humidity and temperature in the THz-TDS system was carefully controlled below $5 \%$ and at $20^{\circ} \mathrm{C}$, respectively, by a dehumidifier and a heater system to prevent $\mathrm{THz}$-absorptions of water vapor [19] and thermal fluctuation of samples.

\subsection{Preparation of Lactose Sample}

Two types of lactose powders purchased from SigmaAldrich Co., Ltd. were used for source materials. The one was $\alpha$-D-lactose monohydrate $\left(\mathrm{L}_{\alpha} \cdot \mathrm{H}_{2} \mathrm{O}: \mathrm{O}-\beta\right.$-galactopyranosyl- $(1 \rightarrow 4)-\alpha$-D-glucopyranose monohydrate $\left.\left(\mathrm{C}_{12} \mathrm{H}_{22} \mathrm{O}_{11} \cdot \mathrm{H}_{2} \mathrm{O}\right)\right)$ and the other was anhydrous $\beta$-D-lactose $\left(\mathrm{L}_{\beta}\right.$ : O- $\beta$-galactopyranosyl-( $\left.1 \rightarrow 4\right)-\beta$-D-glucopyranose $\left.\left(\mathrm{C}_{12} \mathrm{H}_{22} \mathrm{O}_{11}\right)\right)$, in which a hydrogen- and a hydroxylcoordination in the glucose-unit are spatially different in each other as shown in Figure 2. It should be noted here that the lactose powders were including the anomer. The anomer content in the $\mathrm{L}_{\alpha} \cdot \mathrm{H}_{2} \mathrm{O}$ powder (content of $\mathrm{L}_{\beta}$ ) and the $\mathrm{L}_{\beta}$ powder (content of $\mathrm{L}_{\alpha} \cdot \mathrm{H}_{2} \mathrm{O}$ ) were about $4 \%$ and
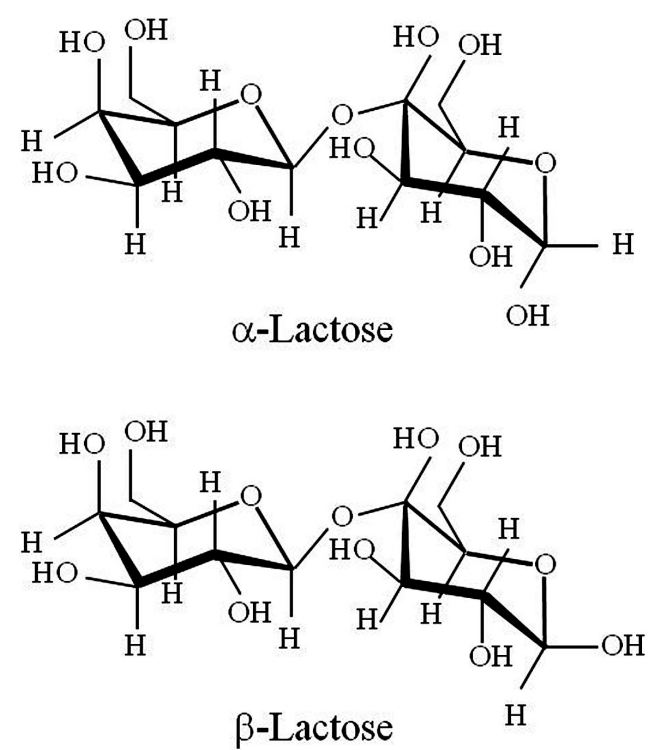

Figure 2. Molecular structures of $\alpha$-D-lactose ( $\alpha$-lactose) and $\beta$-D-lactose ( $\beta$-lactose). 
below $30 \%$ in the commercially specifications, respectively. The lactose powers were mixed with various weight ratios and milled, then filled in a metal aperture with a hole and compressed to form pellet of $6 \mathrm{~mm}$-diameter and $0.7 \mathrm{~mm}$-thickness with parallel surfaces. The pellet was placed with the aperture in the THz-TDS system and THz-wave was normally incident to the pellet surface. After the THz-TDS measurement, weight of the lactose-pellet was measured by an electronic weightscale.

\subsection{Extraction from Lactose Solutions}

Two types of extract processes (refine and unrefined process) were used to examine the extracting behavior of $\mathrm{L}_{\alpha} \cdot \mathrm{H}_{2} \mathrm{O}$ from lactose solution, where the $\mathrm{L}_{\alpha} \cdot \mathrm{H}_{2} \mathrm{O}$ powder was dissolved in ultra-pure water with the resistivity above $18.2 \mathrm{M} \Omega \mathrm{cm}$. As the refine process, lactose was crystallized by seeding of the $\mathrm{L}_{\alpha} \cdot \mathrm{H}_{2} \mathrm{O}$ powder $(10 \mathrm{mg})$ into a supersaturated solution at $20^{\circ} \mathrm{C}\left(1 \mathrm{~g}-\mathrm{L}_{\alpha} \cdot \mathrm{H}_{2} \mathrm{O} / 3\right.$ cc-water) for $24 \mathrm{hrs}$ and dried at $60^{\circ} \mathrm{C}$ for $72 \mathrm{hrs}$ in an incubator after removal the residual solution, then milled and pressed in the aperture. On the other, lactose was crystallized from an unsaturated solution $\left(0.3 \mathrm{~g}-\mathrm{L}_{\alpha} \cdot \mathrm{H}_{2} \mathrm{O} / 3\right.$ cc-water) without the seeding, where water in the solution was gradually vaporized in a dehumidifier for $24 \mathrm{hrs}$ to form wet powder then milled and pressed in the aperture after dry at $60^{\circ} \mathrm{C}$ for $72 \mathrm{hrs}$ in an incubator.

\section{Results and Discussions}

\subsection{Absorption Property of Lactose Powders}

Figures 3 show (a) transmission and (b) absorption spectra of $\mathrm{L}_{\alpha} \cdot \mathrm{H}_{2} \mathrm{O}$ powder (denoted as $\alpha_{\mathrm{P}}$ ) and $\mathrm{L}_{\beta}$ powder $\left(\beta_{\mathrm{P}}\right)$, where the incident THz-wave (Blank) without the lactose sample is also shown in (a). In these spectra, any absorption due to water vapor could not be observed in sufficiently low humidity $(<5 \%)$ and spectrum fluctuation due to aliasing was successfully removed by Gaussian window superposed on the THz-pulse. Since THz-wave from 0.2 to $3 \mathrm{THz}$ could be observed in this system but the intensity was exponentially decreased with the frequency, the frequency useful to analyze the lactose was limited below $1.7 \mathrm{THz}$ because of absorption due to lactose-pellet. Typical narrow-bands and broad-band increasing with frequency were observed in the absorption spectra as shown Figure 3(b). Intense absorptions with peak at 0.53 and $1.37 \mathrm{THz}$ were observed in the $\mathrm{L}_{\alpha} \cdot \mathrm{H}_{2} \mathrm{O}$ powder $\left(\alpha_{\mathrm{P}}\right)$, which were in good agreement to other reports by THzTDS [18] and assigned to lactose-active modes originated from $\mathrm{L}_{\alpha}$-molecular rotations in $\mathrm{L}_{\alpha} \cdot \mathrm{H}_{2} \mathrm{O}$ crystal as shown by first-principles calculations [20]. The absorptions were significantly decreased in the $\mathrm{L}_{\beta} \operatorname{powder}\left(\beta_{\mathrm{P}}\right)$ but the other absorption with peak at peak $1.19 \mathrm{THz}$ was clearly observed. Figure 4 shows the absorption spectra
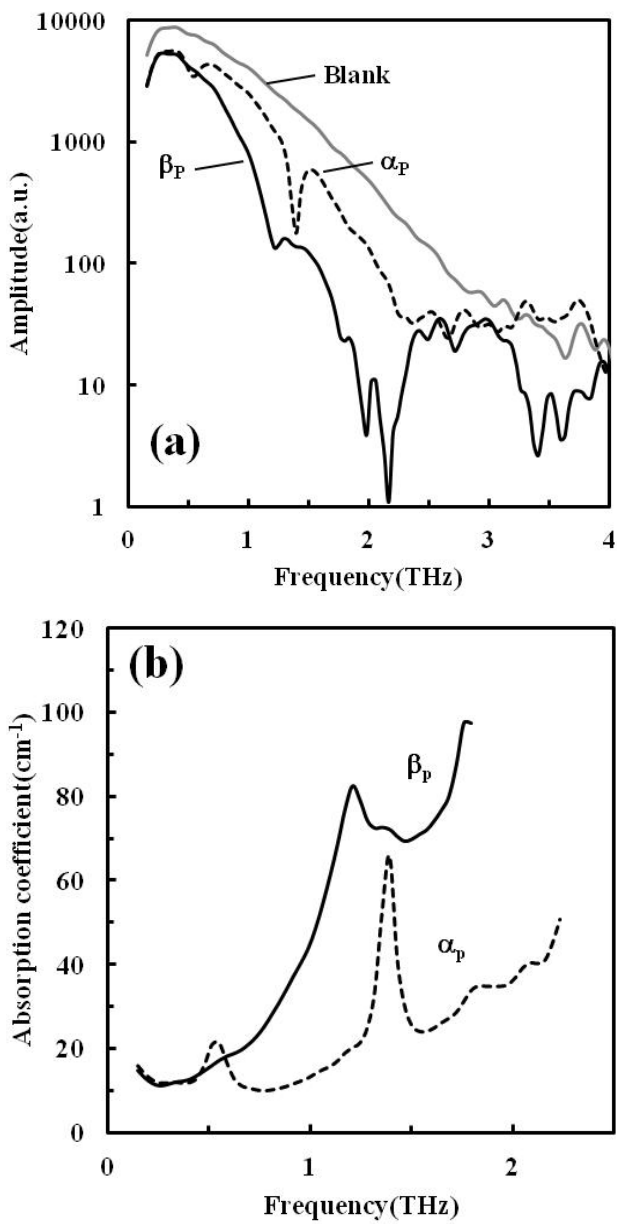

Figure 3. (a) Transmission and (b) absorption spectra of commercially available $\alpha$-D-powder (denoted as " $\alpha_{\mathrm{P}}$ ") and $\beta$-D-lactose powder (denoted as " $\beta_{\mathrm{P}}$ "). The spectrum denoted as "Blank" in (a) was obtained without lactose sample.

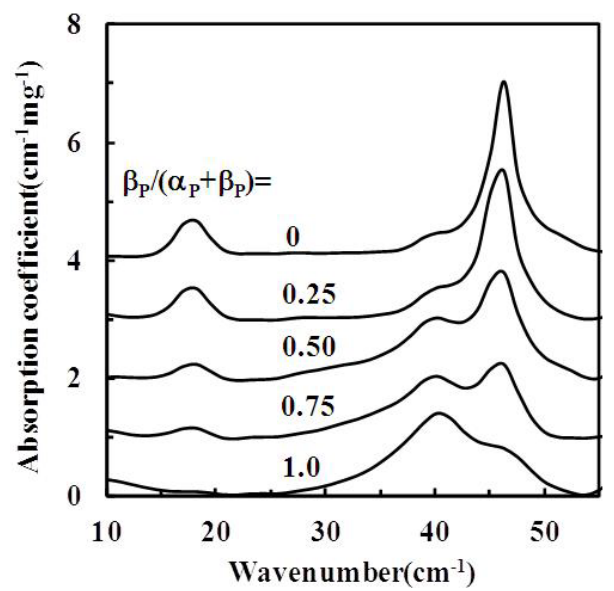

Figure 4. Absorption spectra of the $\alpha$-D-lactose powder and the $\beta$-D-lactose powder mixtures with various $\beta$-D-lactose powder mixed weight-ratio, where the absorption coefficient was normalized by the sample weight in $\mathrm{mg}$ and the background broad-band was removed by polynomial function. 
of lactose-pellets consisting of the $\mathrm{L}_{\alpha} \cdot \mathrm{H}_{2} \mathrm{O}$ powder and $\mathrm{L}_{\beta}$ powder with various weight-content of the $\mathrm{L}_{\beta}$ powder $\left(\beta_{\mathrm{P}} /\left(\alpha_{\mathrm{P}}+\beta_{\mathrm{P}}\right)\right)$ in the pellets, where the broad-band was removed by polynomial cubic function. It is noted that the coefficient should be used in the molar coefficient but was normalized by the weight of pellet because of the uncertain anomer ratio in the $\mathrm{L}_{\alpha} \cdot \mathrm{H}_{2} \mathrm{O}$ powder and the $\mathrm{L}_{\beta}$ powder. The intense absorption with peaks at $17.5 \mathrm{~cm}^{-1}$ $(0.53 \mathrm{THz})$ and $45.6 \mathrm{~cm}^{-1}(1.37 \mathrm{THz})$ observed in the $\mathrm{L}_{\alpha} \cdot \mathrm{H}_{2} \mathrm{O}$ powder were gradually decreased with the $\mathrm{L}_{\beta^{-}}$ content whereas the absorption with peak at $39.7 \mathrm{~cm}^{-1}$ $(1.19 \mathrm{THz})$ was gradually increased with the rate. It is not difficult to recognize from the results that the absorption dominated in $\mathrm{L}_{\beta}$ powder is derived from $\mathrm{L}_{\beta}$. Further, it is expected that the net anomer content in the powders can be evaluated from the intensity ratio of the absorptions derived from $\mathrm{L}_{\alpha} \cdot \mathrm{H}_{2} \mathrm{O}$ and $\mathrm{L}_{\beta}$. For the purpose, the absorption spectra as shown in Figure 4 have to be deconvoluted to each spectrum. Previously, it was reported that the lowest-lying absorption with peak at $17.5 \mathrm{~cm}^{-1}$ observed in $\mathrm{L}_{\alpha} \cdot \mathrm{H}_{2} \mathrm{O}$ powder can be successfully fitted by Lorentzian [21]. In this work, not only the lowest-lying absorption but also the other absorptions below $55 \mathrm{~cm}^{-1}$ were successfully fitted by Lorentzian as shown in Figure 5, where the dot-line depicts a fit by four spectra with peak at 17.5, 30.0, 39.7 and $45.6 \mathrm{~cm}^{-1}$ (solid-lines) with the experimentally obtained absorption for a lactose pellet of $50 \mathrm{wt} \% \mathrm{~L}_{\beta}$ powder (solid-circles). In the spectral feature, the absorption with peak at $39.7 \mathrm{~cm}^{-1}$ due to $\mathrm{L}_{\beta}$ (FWHM around $7.5 \mathrm{~cm}^{-1}$ ) was significantly broader than the two spectra due to $\mathrm{L}_{\alpha} \cdot \mathrm{H}_{2} \mathrm{O}$ (FWHM below $4 \mathrm{~cm}^{-1}$ ). The absorption around $30 \mathrm{~cm}^{-1}$ was also broad with the FWHM of 7.8 and slightly observed in the pellet with the $\mathrm{L}_{\beta}$ powder weight-ratio above $25 \%$, but the absorption was not originated from anhydrous $\mathrm{L}_{\beta}$ as discussed in Section 3.3.

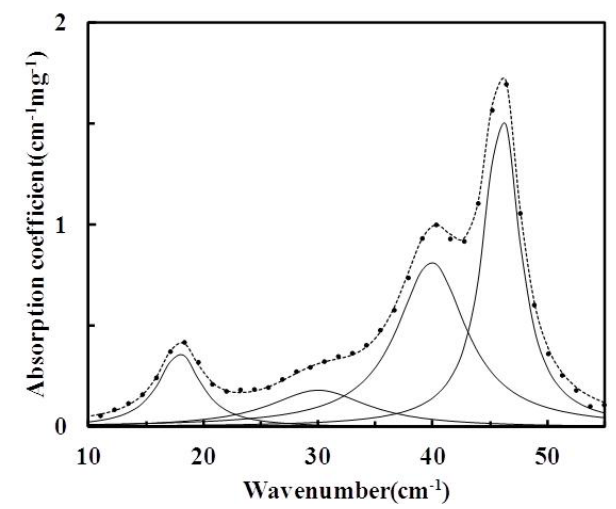

Figure 5. Absorption spectrum of the $\alpha$-D-lactose powder and the $\beta$-D-lactose powder mixture with the $\beta$-D-lactose powder mixed weight-ratio of $50 \%$, where the experimentally obtained data, the deconvoluted spectra and a fit by the spectra are shown by solid-circles, solid-lines and a dotted-line, respectively.

\subsection{Determination of Anomer Content}

The integrated intensities of absorptions with peak at $45.6 \mathrm{~cm}^{-1}$ (open-circles and denoted as " $\mathrm{I}_{\alpha}$ ") and 39.7 $\mathrm{cm}^{-1}$ (closed-circles and denoted as " $\mathrm{I}_{\beta}$ ") were linearly dependent on the $\mathrm{L}_{\beta}$ powder weight-content as shown in Figure 6, in which the correlative square-factors on the least squares method were $99.8 \%$ and $99.6 \%$ for $\mathrm{I}_{\alpha}$ and $\mathrm{I}_{\beta}$ respectively. The results indicate the intensities of $\mathrm{I}_{\alpha}$ and $\mathrm{I}_{\beta}$ for the $\mathrm{L}_{\beta}$ powder weight-ratio can be shown by the below relationships.

$$
\begin{aligned}
\mathrm{I}_{\alpha} & =\mathrm{P}_{\alpha} x_{\alpha} \alpha_{\mathrm{w}}+\mathrm{P}_{\beta}\left(1-x_{\beta}\right) \alpha_{\mathrm{w}} \\
& =-\alpha_{\mathrm{w}}\left(x_{\alpha}+x_{\beta}-1\right) \mathrm{P}_{\beta}+\alpha_{\mathrm{w}} x_{\alpha} \\
& =-\mathrm{r}_{\alpha} \mathrm{P}_{\beta}+\alpha_{\mathrm{w}} x_{\alpha} \\
\mathrm{I}_{\beta} & =\mathrm{P}_{\alpha}\left(1-x_{\alpha}\right) \alpha_{\mathrm{w}}+\mathrm{P}_{\beta} x_{\beta} \beta_{\mathrm{w}} \\
& =\beta_{\mathrm{w}}\left(x_{\alpha}+x_{\beta}-1\right) \mathrm{P}_{\beta}+\beta_{\mathrm{w}}\left(1-x_{\alpha}\right) \\
& =\mathrm{r}_{\alpha} \mathrm{P}_{\beta}+\beta_{\mathrm{w}}\left(1-x_{\alpha}\right)
\end{aligned}
$$

where $\mathrm{P}_{\alpha}$ and $\mathrm{P}_{\beta}$ are mixed weight-ratio of the $\mathrm{L}_{\alpha} \cdot \mathrm{H}_{2} \mathrm{O}$ powder and the $\mathrm{L}_{\beta}$ powder $\left(\mathrm{P}_{\alpha}+\mathrm{P}_{\beta}=1\right), x_{\alpha}$ and $x_{\beta}$ are net anomer weight-content in the $\mathrm{L}_{\alpha} \cdot \mathrm{H}_{2} \mathrm{O}$ powder and the $\mathrm{L}_{\beta}$ powder, $\alpha_{\mathrm{w}}$ and $\beta_{\mathrm{w}}$ are absorption coefficient per weight of $\mathrm{L}_{\alpha} \cdot \mathrm{H}_{2} \mathrm{O}$ and $\mathrm{L}_{\beta}, \mathrm{r}_{\alpha}$ and $\mathrm{r}_{\beta}$ are rate constant of $\mathrm{I}_{\alpha}$ and $\mathrm{I}_{\beta}$ for $\mathrm{L}_{\beta}$ weight-ratio, respectively. Then, the rate constant ratio (r) of $\mathrm{r}_{\alpha} / \mathrm{r}_{\beta}$ for the $\mathrm{L}_{\beta}$-powder weight-content can be reduced from the Equation (1) and (2) to the follow,

$$
r=\frac{r_{\alpha}}{r_{\beta}}=\frac{\alpha_{\mathrm{w}}}{\beta_{\mathrm{w}}}=\frac{360 \alpha_{\mathrm{M}}}{342 \beta_{\mathrm{M}}}=1.05 \frac{\alpha_{\mathrm{M}}}{\beta_{\mathrm{M}}}
$$

where $\alpha_{\mathrm{M}}$ and $\beta_{\mathrm{M}}$ are molar-absorption coefficient of $\mathrm{L}_{\alpha} \cdot \mathrm{H}_{2} \mathrm{O}$ and $\mathrm{L}_{\beta}$, and 360 and 342 are molecular weight of

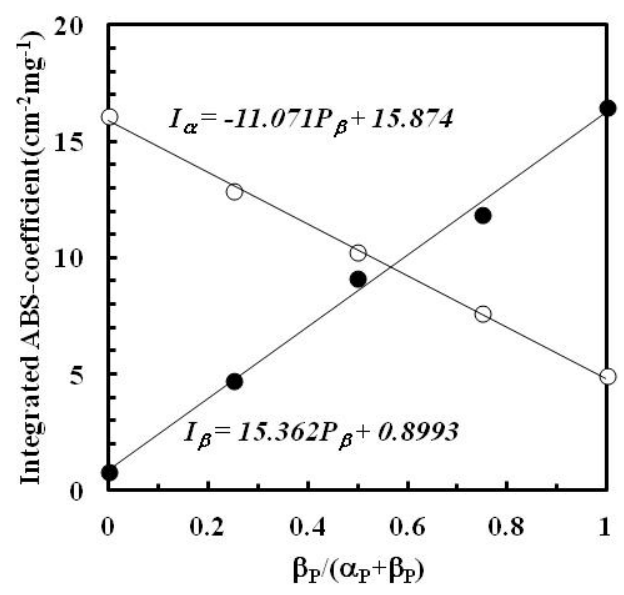

Figure 6. Integrated intensity of absorption derived from $\alpha$-D-lactose monohydrate (peak at $45.6 \mathrm{~cm}^{-1}$; open circles) and anhydrous $\beta$-D-lactose (peak at $39.7 \mathrm{~cm}^{-1}$; solid-circles) for the $\beta$-D-lactose powder mixed weight-ratio in the $\alpha$ - and $\beta$-lactose powders mixture, where the relationships for $I_{\alpha}$ and $I_{\beta}$ were obtained by the least-squares method. 
$\mathrm{L} \alpha \cdot \mathrm{H}_{2} \mathrm{O}$ and $\mathrm{L}_{\beta}$. The rate constant ratio of $\mathrm{r}$ could be determined as 0.721 since the $r_{\alpha}$ and $r_{\beta}$ were experimentally obtained by the results in Figure 6 as 11.07 and 13.36 respectively. On the other, the integrated absorption ratio of $\mathrm{I}_{\alpha}\left(\mathrm{z}_{\alpha}\right)$ and $\mathrm{I}_{\beta}\left(\mathrm{z}_{\beta}\right)$ in lactose-powder including $\mathrm{z}_{\alpha}$-mol $\mathrm{L}_{\alpha} \cdot \mathrm{H}_{2} \mathrm{O}$ and $\mathrm{z}_{\beta}$-mole $\mathrm{L}_{\beta}$ in the $\mathrm{THz}$-wave pathway should be described as below,

$$
\frac{\mathrm{I}_{\beta}\left(\mathrm{z}_{\beta}\right)}{\mathrm{I}_{\alpha}\left(\mathrm{z}_{\alpha}\right)}=\frac{\beta_{\mathrm{w}} \mathrm{z}_{\beta}}{\alpha_{\mathrm{w}} \mathrm{z}_{\alpha}}=\frac{1.05}{\mathrm{r}} \cdot \frac{\mathrm{z}_{\beta}}{\mathrm{z}_{\alpha}} .
$$

As a result, the net mole-ratio $\left(\mathrm{z}_{\beta} / \mathrm{z}_{\alpha}\right)$ in the sample can be determined by the integrated absorption ratio of $\mathrm{I}_{\beta}\left(\mathrm{z}_{\beta}\right)$ / $\mathrm{I}_{\alpha}\left(\mathrm{z}_{\alpha}\right)$ and the r-value $(0.721)$. Figure 7 shows net $\mathrm{L}_{\beta}$ molar-content in the pellets with various $\mathrm{L}_{\beta}$ weight-content evaluated by the Equation (4) using the integrated absorption ratio $\left(\mathrm{I}_{\beta} / \mathrm{I}_{\alpha}\right)$ and the r-value. In the result, correlative square-factors by the least squares method for the dependence on the weight-ratio showed an excellent value as high as $99.8 \%$. The net $\mathrm{L}_{\beta}$ molar-content uniquely increased from $3.9 \%$ to $70.9 \%$ with the $\mathrm{L}_{\beta}$-powder weight-ratio indicated anomer ratio was $3.9 \%$ in the $\mathrm{L}_{\alpha} \cdot \mathrm{H}_{2} \mathrm{O}$ powder and $29.1 \%$ in the $\mathrm{L}_{\beta}$ powder. The anomer ratios in the preliminary used powders were in good agreement to the commercial specifications $\left(\mathrm{L}_{\beta}\right.$ about $4 \%$ in the $\mathrm{L}_{\alpha} \cdot \mathrm{H}_{2} \mathrm{O}$ powder, $\mathrm{L}_{\alpha} \cdot \mathrm{H}_{2} \mathrm{O}$ below $30 \%$ in the $\mathrm{L}_{\alpha} \cdot \mathrm{H}_{2} \mathrm{O}$ powder). The quantitative coincident can be concluded the analysis using the absorptions with peak at $45.6 \mathrm{~cm}^{-1}$ and $39.7 \mathrm{~cm}^{-1}$ were suitable to determine the anomer content in lactose powders. It should be mentioned that the evaluation can be applied to lactose-including samples with uncertain thickness and density because the ratio can be determined by the integrated intensity ratio in the THz-absorption spectrum and the r-value.

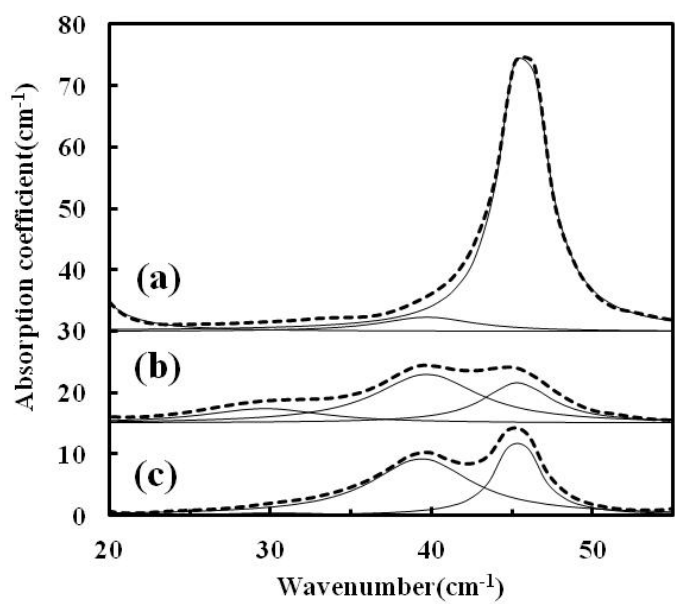

Figure 7. Net anhydrous $\beta$-D-lactose molar-content for the $\beta$-D-lactose powder weight-ratio in the $\alpha$ - and $\beta$-lactose powders mixtures, where the net molar-content in the $\beta$ D-lactose powder $\left(\beta_{\mathrm{P}} /\left(\alpha_{\mathrm{P}}+\beta_{\mathrm{P}}\right)=1\right)$ and the $\alpha$-D-powder $\left(\beta_{\mathrm{P}} /\left(\alpha_{\mathrm{P}}+\beta_{\mathrm{P}}\right)=0\right)$ were evaluated as $71.9 \%$ and $3.9 \%$ respectively.

\subsection{Anomer Content in Extracted Lactose Powders}

As a demonstration to determine the $\alpha$-lactose $/ \beta$-lactose molar-content ratio, lactose powders extracted from lactose solution were examined by THz-TDS as shown in Figure 8, in which the absorption spectra after removal background broad-band and deconvoluted spectra are shown by dot-lines and solid-lines respectively. It is noted that the absorption coefficient is shown in $\mathrm{cm}^{-1}$ without normalization by the lactose-weight. Commonly, it has been recognized that $\mathrm{L}_{\alpha} \cdot \mathrm{H}_{2} \mathrm{O}$ can be crystallized in supersaturated lactose solution [22]. Figure 8(a) shows THz-absorption spectrum of lactose extracted from a supersaturated solution $\left(1.0 \mathrm{~g}-\mathrm{L}_{\alpha} \cdot \mathrm{H}_{2} \mathrm{O}\right.$ powder $/ 3$ cc-water $)$ by seeing of $10 \mathrm{mg}-\mathrm{L}_{\alpha} \cdot \mathrm{H}_{2} \mathrm{O}$ powder including $\mathrm{L}_{\beta}$ with 3.9 $\%$. The lactose was extracted at $20^{\circ} \mathrm{C}$ for $24 \mathrm{hrs}$ and dried at $60^{\circ} \mathrm{C}$ for $72 \mathrm{hrs}$ after removal the solution and then milled and pressed in the aperture. Absorption spectrum consisting of intense $\mathrm{L}_{\alpha} \cdot \mathrm{H}_{2} \mathrm{O}$-absorption and weak $\mathrm{L}_{\beta}$ absorption showed a net $\mathrm{L}_{\beta}$-lactose molar-content as low as $2.8 \%$, which showed the $\mathrm{L}_{\alpha} \cdot \mathrm{H}_{2} \mathrm{O}$ was refined. In contrast, the absorption by lactose powder gradually extracted from lactose solution without the seeding showed quite different behavior as shown in Figures 8(b) and (c), where (a) the water of $3 \mathrm{cc}$ in unsaturated lactose solution $\left(0.3 \mathrm{~g}-\mathrm{L}_{\alpha} \cdot \mathrm{H}_{2} \mathrm{O}\right.$ powder $/ 3$ cc-water $)$ was gradually vaporized at $25^{\circ} \mathrm{C}$ for $24 \mathrm{hrs}$ in a dehumidifier and then (b) dried at $60^{\circ} \mathrm{C}$ for $72 \mathrm{hrs}$. For the spectrum of $\mathrm{L}_{\alpha} \cdot \mathrm{H}_{2} \mathrm{O}$ absorption with peak at $45.6 \mathrm{~cm}^{-1}$, the FWHM was decreased from $5.1 \mathrm{~cm}^{-1}$ to $3.1 \mathrm{~cm}^{-1}$ by the post-anneal. In contrast, any decrease of the FWHM of $\mathrm{L}_{\beta}$-absorption with peak at $39.7 \mathrm{~cm}^{-1}$ was not substantially observed after the post-annealing. The significant difference of the

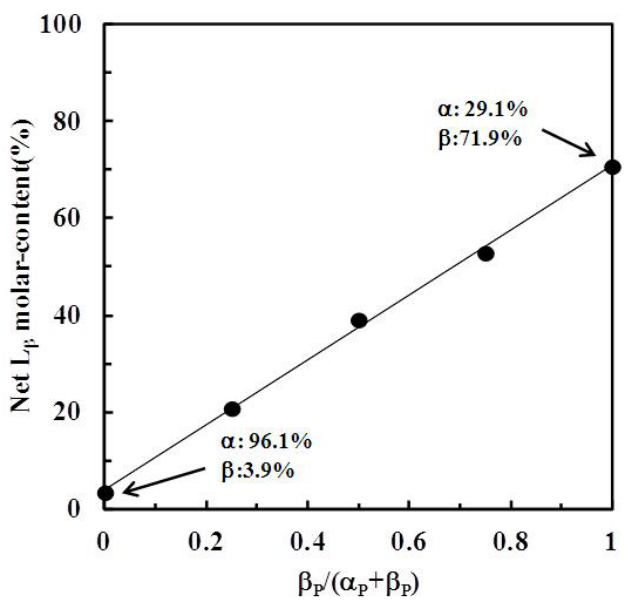

Figure 8. Absorption spectra of (a) refined lactose in a supersaturated lactose-solution, (b) as-extracted lactose by gradual evaporation of water from unsaturated lactose solution and (c) post-annealed lactose of the sample for (b), where the dot-lines and the plain-lines show experimentally obtained spectra and the deconvoluted spectra. 
two absorption behaviors by the post-annealing suggested that $\alpha$-lactose can be crystallized into the hydrates with excess water although anhydrous crystal is formed by $\beta$-lactose. The peak intensity ratio of $\mathrm{L}_{\beta} / \mathrm{L}_{\alpha} \cdot \mathrm{H}_{2} \mathrm{O}$ was decreased but the integrated intensity ratio was increased from $46.2 \%$ to $62.7 \%$ by the post-annealing because the absorption of $\mathrm{L}_{\alpha} \cdot \mathrm{H}_{2} \mathrm{O}$ was narrowed by the annealing. As a result, the net $\mathrm{L}_{\beta}$-molar ratio was increased from $46.2 \%$ to $62.7 \%$ after the annealing.

The evaluated net $\mathrm{L}_{\beta}$-molar ratio of $46.2 \%$ in the asextracted powder was higher than that in the refined powder but significantly low comparing to the equilibrium ratio of $62.7 \%$ in solution [23], which could be recognized by the solubility of $\mathrm{L}_{\alpha}$ lower than of $\mathrm{L}_{\beta}$ [24]. The increased of net $\mathrm{L}_{\beta}$-molar ratio after the post-annealing indicated non-crystallized lactose was crystallized in mostly $\mathrm{L}_{\beta}$ during the annealing. It was reported that collapsed lactose formed from non-collapsed spraydried amorphous lactose by exposure in 50\% RH for long time is crystallized in mostly $\mathrm{L}_{\beta}$ with some presence of $\mathrm{L}_{\alpha} \cdot \mathrm{H}_{2} \mathrm{O}$ at relatively low temperature of $70^{\circ} \mathrm{C}$ [8]. Crystallization of the extracted powder in this work seemed to be similar to the crystallization of collapsed lactose, that is, collapsed lactose was included in the as-extracted lactose powder. It should be mentioned that the broadband around $30 \mathrm{~cm}^{-1}$ observed in the as-dried lactose was almost disappeared after the annealing. The decrease of the absorption and the preference crystallization into $\mathrm{L}_{\beta}$ during the annealing speculates the broad-band is derived from collapsed lactose.

\section{Conclusion}

Transmission-type THz-TDS was applied to non-destructive evaluation of anomer content in lactose powder. Absorption spectra of lactose-pellets consisting of $\mathrm{L}_{\alpha} \cdot \mathrm{H}_{2} \mathrm{O}$ and $\mathrm{L}_{\beta}$ powders were significantly dependent on the mixed rate. The absorption with a peak at $39.7 \mathrm{~cm}^{-1}$ (1.19 THz) was assigned to be originated from $\mathrm{L}_{\beta}$ in addition to the fingerprint of $\mathrm{L}_{\alpha} \cdot \mathrm{H}_{2} \mathrm{O}$ at $17.5 \mathrm{~cm}^{-1}(0.53$ $\mathrm{THz})$ and $45.6 \mathrm{~cm}^{-1}(1.37 \mathrm{THz})$. Since the integrated values of the absorption coefficient were uniquely dependent on the powder mixed rate of $\mathrm{L}_{\alpha} \cdot \mathrm{H}_{2} \mathrm{O} / \mathrm{L}_{\beta}$, the net anomer content in lactose powder could be precisely determined such as $3.9 \%$ and $70.9 \%$ for $\mathrm{L}_{\alpha} \cdot \mathrm{H}_{2} \mathrm{O}$ and $\mathrm{L}_{\beta}$ powders, respectively. Low $\mathrm{L}_{\beta}$-content of $2.8 \mathrm{~mol} \%$ in lactose powders evaluated by the THz-TDS analysis showed the refined feature of $\mathrm{L}_{\alpha} \cdot \mathrm{H}_{2} \mathrm{O}$ in supersaturated lactose solution using $\mathrm{L}_{\alpha} \cdot \mathrm{H}_{2} \mathrm{O}$-seeds. In contrast, $\mathrm{L}_{\beta^{-}}$ content in lactose powder extracted from unsaturated lactose solution by gradual water evaporation was increased from $34.4 \%$ to $45.3 \%$ by post-annealing at $60^{\circ} \mathrm{C}$. A broad-band with a peak at $30.5 \mathrm{~cm}^{-1}$ was speculated to collapsed-lactose absorption by the behavior crystallized in mostly $\mathrm{L}_{\beta}$ after the annealing.

\section{Acknowledgements}

This research was partially supported by JSPS KAKENHI Grant-in-Aid for Exploratory Research Number 24655165.

\section{REFERENCES}

[1] M. G. Gänzle, G. Haase and P. Jelen, "Lactose: Crystallization, Hydrolysis and Value-Added Derivatives," International Dairy Journal, Vol. 18, No. 7, 2008, pp. 685695. http://dx.doi.org/10.1016/j.idairyj.2008.03.003

[2] C. S. Hudson, "Future Studies on the Forms of MilkSugar," Journal of the American Chemical Society, Vol. 30, No. 11, 1908, pp. 1767-1783.

http://dx.doi.org/10.1021/ja01953a015

[3] C. A. Aguilar and G. R. Ziegler, "Physical and Microscopic Characterization of Dry Whole Milk with Altered Lactose Content. 2. Effect of Lactose Crystallization," Journal of Dairy Science, Vol. 77, No. 5, 1994, pp. 11981204.

http://dx.doi.org/10.3168/jds.S0022-0302(94)77058-2

[4] P. Würsch, J. Rosset, B. Köllreutter and A. Klein, "Crystallization of $\beta$-Lactose under Elevated Storage Temperature in Spray-Dried Milk Powder," Milchwissenschaft, Vol. 39, No. 10, 1984, pp. 579-582.

[5] K. Jouppila, J. Kansikas and Y. H. Roos, "Glass Transition, Water Plasticization, and Lactose Crystallization in Skim Milk Powder," Journal of Dairy Science, Vol. 80, No. 12, 1997, pp. 3152-3160. http://dx.doi.org/10.3168/jds.S0022-0302(97)76286-6

[6] E. Berlin, B. A. Anderson and M. J. Pallansch, "Water Vapor Sorption Properties of Various Dried Milks and Whey," Journal of Dairy Science, Vol. 51, No. 9, 1968, pp. 1339-1344.

http://dx.doi.org/10.3168/jds.S0022-0302(68)87191-7

[7] N. Drapier-Beche, J. Fanni, M. Parmentier and M. Vilasi, "Evaluation of Lactose Crystalline Forms by Nondestructive Analysis," Journal of Dairy Science, Vol. 80, No. 3, 1997, pp. 457-463.

http://dx.doi.org/10.3168/jds.S0022-0302(97)75957-5

[8] P. Darcy and G. Buckton, "The Influence of Heating: Drying on the Crystallisation of Amorphous Lactose after Structural Collapse," International Journal of Pharmaceutics, Vol. 158, No. 2, 1997, pp. 157-164. http://dx.doi.org/10.1016/S0378-5173(97)00245-7

[9] Á. GombÁs, P. Szabó-Révész, M. Kata, J. G. Regdon and I. Erős, "Quantitative Determination of Crystallinity of $\alpha$-Lactose Monohydrate by DSC," Journal of Thermal Analysis and Calorimetry, Vol. 68, No. 2, 2002, pp. 503510. http://dx.doi.org/10.1023/A:1016039819247

[10] B. M. Murphya, S. W. Prescottb and I. Larson, "Measurement of Lactose Crystallinity Using Raman Spectroscopy," Journal of Pharmaceutical and Biomedical Analysis, Vol. 38, No. 1, 2005, pp. 186-190.

http://dx.doi.org/10.1016/j.jpba.2004.12.013

[11] J. H. Kirk, S. E. Dann and C. G. Blatchford, "Lactose: A Definitive Guide to Polymorph Determination," International Journal of Pharmaceutics, Vol. 334, No. 1-2, 2007, pp. 103-114. 
http://dx.doi.org/10.1016/j.ijpharm.2006.10.026

[12] Md. K. Haque and Y. H. Roos, "Lactose: A Definitive Guide to Polymorph Determination," Carbohydrate Research, Vol. 340, No. 2, 2005, pp. 293-301.

[13] M. Yamaguchi, F. Miyamaru, K. Yamamoto, M. Tani and M. Hangyo, "Terahertz Absorption Spectra of L-, D-, and DL-Alanine and Their Application to Determination of Enantiometric Composition," Applied Physics Letters, Vol. 86, 2005, Article ID: 053903. http://dx.doi.org/10.1063/1.1857080

[14] F. Miyamaru, M. Yamaguchi, M. Tani, M. Hangyo, K. Yamamoto and K. Tominaga, "THz-Time-Domain Spectroscopy of Amino Acids in Solid Phase," Conference on Lasers and Electro-Optics, Baltimore, 6 June 2003.

[15] M. Walther, B. Fischer, M. Schall, H. Helm and P. U. Jepsen, "Far-Infrared Vibrational Spectra of All-Trans, 9-cis and 13-cis Retinal Measured by THz Time-Domain Spectroscopy," Chemical Physics Letters, Vol. 332, No. 3-4, 2000, pp. 389-395. http://dx.doi.org/10.1016/S0009-2614(00)01271-9

[16] P. C. Upadhya, Y. C. Shen, A. G. Davies and E. H. Linfield, "Far-Infrared Vibrational Modes of Polycrystalline Saccharides," Vibrational Spectroscopy, Vol. 35, No. 1, 2004, pp. 139-143. http://dx.doi.org/10.1016/j.vibspec.2003.12.010

[17] H. B. Liu and X. C. Zhang, "Dehydration Kinetics of DGlucose Monohydrate Studied Using THz Time-Domain Spectroscopy," Chemical Physics Letters, Vol. 429, No. 1-3, 2006, pp. 229-233. http://dx.doi.org/10.1016/j.cplett.2006.07.100

[18] A. Roggenbuck, H. Schmitz, A. Deninger, I. C. Mayorga, J. Hemberger, R. Güsten and M. Grüninger, "Coherent Broadband Continuous-Wave Terahertz Spectroscopy on Solid-State Samples," New Journal of Physics, Vol. 12,
2010, Article ID: 043017 http://dx.doi.org/10.1088/1367-2630/12/4/043017

[19] H. M. Pickett, R. L. Poynter, E. A. Cohen and M. L. Delisky, J. C. Pearson and H. S. P. Müller, "Submillimeter Millimeter, and Microwave Spectral Line Catalog," Journal of Quantitative Spectroscopy and Radiative Transfer, Vol. 60, No. 5, 1998, pp. 883-890. http://dx.doi.org/10.1016/S0022-4073(98)00091-0

[20] S. Saito, T. M.Inerbaev, H. Mizuseki, N. Igarashi, R. Note and Y. Kawazoe, "First Principles Calculation of Terahertz Vibrational Modes of a Disaccharide Monohydrate Crystal of Lactose," Japanese Journal of Applied Physics, Vol. 45, No. 43, 2006, pp. L1156-L1158. http://dx.doi.org/10.1143/JJAP.45.L1156

[21] D. G. Allis, A. M. Fedor, T. M. Korter, J. E. Bjarnason and E. R. Brown, "Assignment of the Lowest-Lying THz Absorption Signatures in Biotin and Lactose Monohydrate by Solid-State Density Functional Theory," Chemical Physics Letters, Vol. 440, No. 4-6, 2007, pp. 203-209. http://dx.doi.org/10.1016/j.cplett.2007.04.032

[22] N. Drapier-Beche, J. Fanni and M. Parmentier, "Physical and Chemical Properties of Molecular Compounds of Lactose," Journal of Dairy Science, Vol. 82, No. 12, 1999, pp. 2558-2563. http://dx.doi.org/10.3168/jds.S0022-0302(99)75510-4

[23] T. A. Nickerson, "Lactose Crystallization in Ice Cream. IV. Factors Responsible for Reduced Incidence of Sandiness," Journal of Dairy Science, Vol. 45, No. 3, 1962, pp. 354-359. http://dx.doi.org/10.3168/jds.S0022-0302(62)89398-9

[24] P. Walstra, J. T. Wouters and T. J. Geurts, "Dairy Science and Technology," 2nd Edition, CRC Press, 2005, p. 754. http://dx.doi.org/10.1201/9781420028010.ax7 